\title{
Complete Genome Sequence of Bacillus sp. strain WR11, an Endophyte Isolated from Wheat Root Providing Genomic Insights into Its Plant Growth-Promoting Effects
}

\author{
Can Chen, ${ }^{1}$ Zonghao Yue, ${ }^{1}$ Cuiwei Chu, ${ }^{1}$ Keshi Ma, ${ }^{1}$ Lili Li, ${ }^{2, \dagger}$ and Zhongke Sun ${ }^{1,2, \dagger}$ \\ ${ }^{1}$ College of Life Sciences and Agronomy, Zhoukou Normal University, 466001, China \\ ${ }^{2}$ Key Laboratory of Plant Genetics and Molecular Breeding, Zhoukou Normal University, 466001, China
}

\begin{abstract}
Bacillus sp. strain WR11 isolated from the root of wheat (Triticum aestivum L.) possesses abiotic stress alleviating properties and produces several types of enzymes. However, its genomic information is lacking. The study described the complete genome sequence of the bacterium. The size of the genome was 4202080 base pairs that consisted of 4405 genes in total. The $\mathrm{G}+\mathrm{C}$ content of the circular genome was $43.53 \%$ and there were 4170 coding genes, 114 pseudo genes, 30 ribosome RNAs, 86 tRNAs, and 5 ncRNAs, based on the Prokaryotic Genome Annotation Pipeline (PGAP). Many genes were related to the stressalleviating properties and 124 genes existed in the CAZy database. The complete genome data of strain WR11 will provide valuable resources for genetic dissection of its plant growthpromoting function and symbiotic interaction with plant.
\end{abstract}

\section{Genome Announcement}

As a somewhat inseparable part of wheat, endophytes have important roles for wheat growth and health (Kandel et al. 2017; Khare et al. 2018). These symbiotic microorganisms have multiple growth-promoting effects, including nitrogen fixation, phosphorus dissolving, prevention of pathogens, and acquisition of nutrients, especially under abiotic or biotic stresses (Abd El-Daim et al. 2018; Shankar Naik 2019). The genus Bacillus can be widely isolated from wheat, either as rhizobacteria or as endophytes (Singh et al. 2018). In our previous work, Bacillus sp. strain WR11 was isolated from the root of Triticum aestivum $\mathrm{L}$. (cultivar Zhoumai 26) in China, as described elsewhere (Sun et al. 2017). Strain WR11 showed similar abiotic stress-alleviating properties in wheat seedlings to Bacillus altitudinis WR10 (Yue et al. 2019). However, strain WR11 has a wider enzyme profile than WR10. To provide genomic insights into its abiotic stress alleviating properties, we here describe its complete genome as a valuable resource.

Isolate WR11 was propagated normally in nutrient broth by shaking at $150 \mathrm{rpm}$ overnight at $30^{\circ} \mathrm{C}$. It was identified as a Bacillus species primarily by alignment of the $16 S$ rRNA (ribosome RNA) gene amplified with the bacterial universal primers (27F/1492R) as described (Liu et al. 2018). Bacterial genomic DNA was extracted, using a genomic DNA isolation kit following the manufacturer protocol (CoWin Biosciences, Beijing). The harvested DNA was detected by the agarose gel electrophoresis and was quantified by Qubit assays. Insert libraries were prepared from high-quality genomic DNA of strain WR11, using two standard

\section{${ }^{\dagger}$ Corresponding authors: Z. Sun; sunzh@ daad-alumni.de and L. Li; $13672165360 @ 163 . c o m$}

The author(s) declare no conflict of interest.

Accepted for publication 29 March 2020.

(C) 2020 The American Phytopathological Society

\section{Funding}

This work was partially supported by grants from the Science and Technology Agency of Henan Province (grant number 192102310493) and the China Postdoctoral Science Foundation (grant number 2018M642772).

\section{Keywords}

bacteria-wheat interaction, Bacillus, complete genome, endophytes, plant growth-promoting effects 
Table 1. Genome assembly statistics of Bacillus sp. strain WR11 and its two closest strains

\begin{tabular}{llll} 
& \multicolumn{3}{c}{ Bacillus sp. strain } \\
\cline { 2 - 4 } Parameter & \multicolumn{1}{c}{ WR11 } & YP1 & DM2 \\
NCBI accession & CP033064 & CP010014 & CP030937 \\
Genome size $(\mathrm{Mb})$ & 4.20208 & 4.15357 & 4.32287 \\
Symmetric identity $(\%)$ & 100 & 97.24 & 97.17 \\
GC content (\%) & 43.50 & 43.63 & 43.34 \\
Number of plasmid & 0 & 1 & 1 \\
Number of total genes & 4,405 & 4,136 & 4,527 \\
Number of coding genes & 4,170 & 3,955 & 4,311 \\
\hline
\end{tabular}

protocols (10-kb single molecule real-time [SMRT] bell library and 350-bp Illumina library). The genome was sequenced, using SMRT technology based on the PacBio system, by a commercial supplier (Novogene Bioinformatics Technology Co., Ltd., Beijing). Sequencing yielded 331,927 reads including 2,496,715,606 bp with a mean concordance of 0.85 and the $\mathrm{N}_{50}$ read length of $8,853 \mathrm{bp}$, which corresponds to approximately $594 \times$ sequence depth. The low-quality reads were filtered by SMRT Link v5.0.1 (PacBio software downloads page), and the high-quality reads were assembled to generate one contig without gaps (Ardui et al. 2018). The assembled contig was then optimized and mis-incorporated bases were corrected using the Arrow algorithm (GitHub). After that, a polished contig of 4,202,080 bp was finalized as the circular chromosome and deposited in the National Center for Biotechnology Information (NCBI) database under accession number CP033064.1.

The GeneMarkS program (version 4.17) was used to retrieve the related coding gene (Besemer et al. 2001). Transfer RNA (tRNA) genes were predicted by the tRNAscan-SE (version 1.3.1) (Lowe and Eddy 1997). rRNA genes were analyzed by the rRNAmmer (version 1.2) (Lagesen et al. 2007). Small nuclear RNAs were predicted by BLAST against the Rfam database and were then identified by software Cmsearch (version 1.1rc4) (Nawrocki et al. 2015). All predicted genes were annotated using blastp (BLAST 2.2.28+) against four databases, namely, the Nonredundant (NR) database, Kyoto Encyclopedia of Genes and Genomes (KEGG), clusters of orthologous groups (COG v9.05), and Gene Ontology (GO) databases, through blastp, blastx/blastp, and blast2go. The interspersed repetitive sequences were predicted using RepeatMasker (version open-4.0.5) (Saha et al. 2008). The tandem repeats were analyzed by the Tandem Repeats Finder, version 4.07b (Benson 1999). Automatic annotation was performed as well, using the Prokaryotic Genome Annotation Pipeline (PGAP) ver. 4.2 (Tatusova et al. 2016) during submission into GenBank.

The GeneMarkS program prediction yielded 4,420 genes with an average gene length of $847 \mathrm{bp}$, searching the NR database. This number is slightly higher than the 4,405 genes and 4,170 coding sequences predicted by PGAP. In addition, 4,276, 3,188, and 2,866 genes were annotated in the KEGG, COG, and GO databases, respectively. The KEGG analysis suggested a large number of $A B C$ transporters (145 genes) and two-component systems (134 genes) in WR11. Meanwhile, considerable numbers of genes involved in quorum sensing (80 genes) and biofilm formation (14 genes), which were important for cross-kingdom communication and microbe-plant interaction (Schikora et al. 2016; Kan et al. 2017; Zúñiga et al. 2017), were also found. Furthermore, 1,612 genes in the biological process fell into the category of metabolic process according to the GO analysis. In the ontology of molecular function, 1,534 genes were identified to be of catalytic activity. The fact that so many genes are taking part in the process of metabolism and catalysis suggests that strain WR11 may possess high potential for producing complex and diverse metabolites. Additionally, 1,274 genes were predicted to be associated with molecular function of binding, which may contribute to its plant colonization (Zeng et al. 2020). Furthermore, there were $30 \mathrm{rRNAs}, 86$ tRNAs, and five noncoding RNAs (ncRNAs), in the genome based on the PGAP, albeit a slight difference in numbers of tRNAs and ncRNAs were determined by different methods. There were 271 interspersed nuclear elements and 60 tandem repeats. These repeats with sizes of 7 to 282 bp totally accounted for about $0.63 \%$ in genome. In the NCBI genome database, WR11 has the highest symmetric identity of $>97 \%$ to two other strains that were completely sequenced (Bacillus sp. strain YP1 and Bacillus sp. strain DM2). Therefore, genome assembly statistics of them were summarized for comparison (Table 1). 
As mentioned above, Bacillus sp. strain WR11 has a wider enzyme profile and many of them responded for the stress-alleviating properties. For example, enzymatic activities of 1aminocyclopropane-1-carboxylate (ACC) deaminase and phytase were detected in the culture supernatant of both WR10 and WR11, while genes were not found on the reference genome of $B$. altitudinis GR-8, which has $99.50 \%$ identity in marker gene gyrB to strain WR10 (accession number KY416927.1). In contrast, both potential ACC deaminase (locus_tag D9C22_12100) and phytase (locus_tag D9C22_10655) genes are presented on the genome of strain WR11. Moreover, we predicted carbohydrate-active enzymes (CAZy) by searching the CAZy database (Cantarel et al. 2009). There were as many as 124 genes in the WR11 genome in the CAZy database. The majority enzymes were glycoside hydrolases and glycosyl transferases (57 and 44, respectively). In addition, 15 and six genes were classified as carbohydrate esterases and polysaccharide lyases. Many of them, e.g., cellulase, chitinase, and pectate lyase may involve its colonization and interaction with plants (Alimisup et al. 2012; Kovtunovych et al. 1999; Mota et al. 2017). Other enzymes related to plant growth-promoting properties were searched, using keywords based on the genome annotation information. In particular, enzymes related to the high-salt stress-alleviating property-like catalase and amylase and enzymes related to the low-phosphorus stressalleviating property-like phosphatases were identified on the genome of WR11. All these genes provided important genetic insights into its plant growth-promoting effect, especially the abiotic stress-alleviating properties of strain WR11 on its host plant.

Glycerol stocks of Bacillus sp. strain WR11 are available for academic purpose upon request from $Z$. Sun. The complete genome sequence of WR11 has been deposited in GenBank under accession number CP033064. The version described in this paper is version CP033064.1.

\section{Author-Recommended Internet Resources}

COG database: https://string-db.org/cgi/input.pl

GeneMarkS gene prediction programs: http://topaz.gatech.edu/GeneMark

Gene Ontology: http://www.geneontology.org

GitHub Arrow: https://github.com/PacificBiosciences/GenomicConsensus

KEGG database: https://www.genome.jp/kegg

RepeatMasker: http://www.repeatmasker.org

PacBio software downloads page: https://www.pacb.com/support/software-downloads/

\section{Literature Cited}

Abd El-Daim, I. A., Bejai, S., Fridborg, I., and Meijer, J. 2018. Identifying potential molecular factors involved in Bacillus amyloliquefaciens 5113 mediated abiotic stress tolerance in wheat. Plant Biol Stuttg 20:271-279.

Alimisup, M., Soleimani, M. J., and Darzi, M. T. 2012. Characterization and application of microbial antagonists for control of fusarium head blight of wheat caused by Fusarium graminearum using single and mixture strain of antagonistic bacteria on resistance and susceptible cultivars. Afr. J. Microbiol. Res. 6:6-23.

Ardui, S., Ameur, A., Vermeesch, J. R., and Hestand, M. S. 2018. Single molecule real-time (SMRT) sequencing comes of age: Applications and utilities for medical diagnostics. Nucleic Acids Res. 46:2159-2168.

Benson, G. 1999. Tandem repeats finder: A program to analyze DNA sequences. Nucleic Acids Res. 27:573-580.

Besemer, J., Lomsadze, A., and Borodovsky, M. 2001. GeneMarkS: A self-training method for prediction of gene starts in microbial genomes. Implications for finding sequence motifs in regulatory regions. Nucleic Acids Res. 29:2607-2618.

Cantarel, B. L., Coutinho, P. M., Rancurel, C., Bernard, T., Lombard, V., and Henrissat, B. 2009. The Carbohydrate-Active EnZymes database (CAZy): An expert resource for glycogenomics. Nucleic Acids Res. 37 (Database): D233-D238.

Kan, J., Fang, R., and Jia, Y. 2017. Interkingdom signaling in plant-microbe interactions. Sci. China Life Sci. 60:785-796.

Kandel, S. L., Joubert, P. M., and Doty, S. L. 2017. Bacterial endophyte colonization and distribution within plants. Microorganisms 5:77.
Khare, E., Mishra, J., and Arora, N. K. 2018. Multifaceted interactions between endophytes and plant: Developments and prospects. Front. Microbiol. 9:2732.

Kovtunovych, G., Lar, O., Kamalova, S., Kordyum, V., Kleiner, D., and Kozyrovska, N. 1999. Correlation between pectate lyase activity and ability of diazotrophic Klebsiella oxytoca VN 13 to penetrate into plant tissues. Plant Soil 215:1-6.

Lagesen, K., Hallin, P., Rødland, E. A., Staerfeldt, H. H., Rognes, T., and Ussery, D. W. 2007. RNAmmer: Consistent and rapid annotation of ribosomal RNA genes. Nucleic Acids Res. 35:3100-3108.

Liu, K., He, L., Li, S., Tian, F., Sun, Z., and Li, C. 2018. Draft genome sequence of Paenibacillus strain LK1, a phytohormone producing bacterium. 3 Biotech 8:85.

Lowe, T. M., and Eddy, S. R. 1997. tRNAscan-SE: a program for improved detection of transfer RNA genes in genomic sequence. Nucleic Acids Res. 25:955-964.

Mota, M. S., Gomes, C. B., Souza Júnior, I. T., and Moura, A. B. 2017. Bacterial selection for biological control of plant disease: Criterion determination and validation. Braz. J. Microbiol. 48:62-70.

Nawrocki, E. P., Burge, S. W., Bateman, A., Daub, J., Eberhardt, R. Y., Eddy, S. R., Floden, E. W., Gardner, P. P., Jones, T. A., Tate, J., and Finn, R. D. 2015. Rfam 12.0: Updates to the RNA families database. Nucleic Acids Res. 43(D1): D130-D137.

Saha, S., Bridges, S., Magbanua, Z. V., and Peterson, D. G. 2008. Empirical comparison of ab initio repeat finding programs. Nucleic Acids Res. 36 : 2284-2294.

Schikora, A., Schenk, S. T., and Hartmann, A. 2016. Beneficial effects of bacteriaplant communication based on quorum sensing molecules of the $\mathrm{N}$-acyl homoserine lactone group. Plant Mol. Biol. 90:605-612. 
Shankar Naik, B. 2019. Functional roles of fungal endophytes in host fitness during stress conditions. Symbiosis 79:99-115.

Singh, D., Geat, N., Rajawat, M. V. S., Prasanna, R., Kar, A., Singh, M. S., and Saxena, A. K. 2018. Prospecting endophytes from different Fe or $\mathrm{Zn}$ accumulating wheat genotypes for their influence as inoculants on plant growth, yield, and micronutrient content. Ann. Microbiol. 68:815-833.

Sun, Z., Liu, K., Zhang, J., Zhang, Y., Xu, K., Yu, D., Wang, J., Hu, L., Chen, L., and Li, C. 2017. IAA producing Bacillus altitudinis alleviates iron stress in Triticum aestivum $\mathrm{L}$. seedling by both bioleaching of iron and up-regulation of genes encoding ferritins. Plant Soil 419:1-11.

Tatusova, T., DiCuccio, M., Badretdin, A., Chetvernin, V., Nawrocki, E. P., Zaslavsky, L., Lomsadze, A., Pruitt, K. D., Borodovsky, M., and Ostell, J. 2016. NCBI prokaryotic genome annotation pipeline. Nucleic Acids Res. 44:6614-6624.
Yue, Z., Shen, Y., Chen, Y., Liang, A., Chu, C., Chen, C., and Sun, Z. 2019. Microbiological insights into the stress-alleviating property of an endophytic Bacillus altitudinis WR10 in wheat under low-phosphorus and high-salinity stresses. Microorganisms 7:508.

Zeng, T., Rodriguez-Moreno, L., Mansurkhodzaev, A., Wang, P., van den Berg, W., Gasciolli, V., Cottaz, S., Fort, S., Thomma, B. P. H. J., Bono, J. J., Bisseling, T., and Limpens, E. 2020. A lysin motif effector subverts chitin-triggered immunity to facilitate arbuscular mycorrhizal symbiosis. New Phytol. 225: 448-460.

Zúñiga, A., Donoso, R. A., Ruiz, D., Ruz, G. A., and González, B. 2017. Quorumsensing systems in the plant growth-promoting bacterium Paraburkholderia phytofirmans PsJN exhibit cross-regulation and are involved in biofilm formation. Mol. Plant-Microbe Interact. 30:557-565. 